\title{
The Kalman-Tran-D'Souza Model and the Semileptonic Decay Rates of Heavy Baryons *
}

\author{
I. D'Souza ${ }^{(a)}$, C. S. Kalman ${ }^{(a)}$, P. Yu Kulikov ${ }^{(b)}$ and I. M. Narodetskii ${ }^{(b)}$ \\ ${ }^{(a)}$ Concordia University, Montreal, Canada \\ ${ }^{(b)}$ Institute of Theoretical and Experimental Physics, Moscow, Russia
}

We present an investigation of the inclusive semileptonic decay widths of the heavy baryons $\Lambda_{Q}$ and $\Xi_{Q}(Q=b, c)$ performed within a relativistic constituent quark model, formulated on the light-front. In a way conceptually similar to the deep-inelastic scattering case, the $H_{Q}$-baryon inclusive width is expressed as the integral of the free $Q$-quark partial width multiplied by a bound-state factor related to the $Q$-quark distribution function in the $H_{Q}$. The non-perturbative meson structure is described through the quark-model wave functions, constructed via the Hamiltonian light-front formalism using as input the Kalman-Tran-D'Souza equal time wave functions. A link between spectroscopic quark models and the $H_{Q}$ decay physics is obtained in this way. It is shown that the bound-state effects and the Fermi motion of the $b$-quark remarkably reduce the decay rate with respect to the free-quark result. Our predictions for the $\operatorname{BR}\left(\Lambda_{c} \rightarrow X_{s} \ell \nu_{\ell}\right)$ and $\operatorname{BR}\left(\Lambda_{b} \rightarrow X_{c} \ell \nu_{\ell}\right)$ decays are in good agreement with existing data.

\section{Introduction}

The investigation of inclusive semileptonic $\Lambda_{b}$ decays can provide relevant information on the CabibboKobayashi-Maskawa $(C K M)$ parameters $\left|V_{c b}\right|$ and $\left|V_{u b}\right|$ as well as on the internal non-perturbative structure of the $\Lambda_{b}$ baryon. The first evidence of semileptonic $\Lambda_{b}$ decays had been reported by ALEPH and OPAL collaborations who had seen an excess of correlated $\Lambda_{s} \ell^{-}$pairs over $\Lambda_{s}^{+}$pairs from $Z$ decays [1], [2]. More recently, the CDF collaboration [3] measured the $\Lambda_{b}$ lifetime using its semileptonic decay mode. It is therefore important to study theoretical models for the semileptonic $\Lambda_{b}$ decays.

As far as the theoretical point of view is concerned, the $Q C D$-based operator product expansion $(O P E)$ combined with the heavy quark expansion is widely recognized as a consistent dynamical approach for investigating inclusive heavy-flavour decays [ 4 . Existing theoretical predictions for inclusive weak decays are in reasonable agreement, within the expected range of uncertainty, with the data on decays of charmed and beauty particles. However, the result of the OPE approach for the $\Lambda_{b}$ lifetime is puzzling because it predicts that $\left(\tau_{\Lambda_{b}} / \tau_{B}\right)_{O P E}=$ $0.98+\mathcal{O}\left(1 / m_{b}^{2}\right)$, whereas the experimental finding suggest a very much reduced fraction $\left(\tau_{\Lambda_{b}} / \tau_{B}\right)_{\text {exp }}=$ $0.8 \pm 0.04$. It is not clear whether the present contradiction between the theory and the data on $\tau_{\Lambda_{b}} / \tau_{B}$

\footnotetext{
${ }^{*}$ The invited talk presented by C.S.K. at the $4^{\text {th }}$ International Conference on Hyperons, Charm and Beauty Hadrons, Valencia, June $27-30,2000$. The work by P.Yu.K. and I.M.N. was supported in part by the RFBR grants, ref. No 00-02-16363 and $00-15-96786$
}

is a temporary difficulty, or evidence for the fundamental flaws in the OPE approach. In spite of great efforts of theoretical activity the $\Lambda_{b}$ lifetime remains significantly low which continues to spur theoretical activity. In this respect, the use of phenomenological models, like the constituent quark model, could be of great interest as a complementary approach to the $O P E$ method.

What criteria should be used in determining an appropriate constituent quark model for the baryons? There is now a vast amount of data on the spectrum of baryons. Many quark model calculations of the spectrum attempt to account for the masses, spin etc., without studying the decays. A serious drawback to this approach is that it is difficult to assign predicted baryons to experimentally known particles on the basis of spectrum alone. In this sense this paper is a continuation of the work of Kalman and Tran [5] to produce a complete picture of the spectrum and decays of the baryons. This model is based upon the following principles: i) consistency with the model of the mass calculation - A change of some feature of the mass calculation in the decay model may compromise the purpose; ii) The decay calculation should be mainly determined by the mass calculation. For instance, if there are too many parameters in the decay model it is difficult to relate the results obtained to the particles considered in the model, used in the mass calculation. The decay calculations were based upon the quark pair creation model of Le Yaouanc et. al. [6] They studied the decay processes $N, \Delta \rightarrow N \pi$ and $\Lambda, \Sigma \rightarrow N \bar{K}$. The pair creation strength $\gamma$ is replaced by $\mathrm{k}^{\gamma}$. Besides 
the meson radius, these are the only parameters of the model. The results are in good agreement with the data. To calculate decay widths in their model, Kalman and Tran had to re-correct the originally calculated parameters $\alpha_{\rho}$ and $\alpha_{\lambda}$ by approximately 0.05 $\mathrm{GeV}$ from the values determined from their spectrum calculation, in order to make the decay widths match experimental data. The resulting change in $\alpha_{\rho}$ and $\alpha_{\lambda}$ causes a shift in the originally calculated mass spectrum. It is these values that must be used if the decay widths are to be correct.

At the time that the Kalman-Tran model was introduced, there was little data available for the $b$ and $c$ sectors. The model cannot fit these sectors properly without the modifications performed in the Kalman and D'Souza model [13]. The essential modification is that the coupling constant of the interaction is made to be scale dependent in a manner that does not require knowledge of the behaviour at low energy scales and does not introduce any new parameter. The calculations of the inclusive semileptonic widths and the corresponding branching ratios found here introduce no new parameters. It is based solely upon the eigenfunctions for baryon states found by Kalman and D'Souza [13

In what follows we assume that, instead of QCD with its complicated dynamics of infinite number of degrees of freedom in the light cloud, we consider a constituent bound-state problem of a heavy quark interacting with a lighter ones via a potential. Then, using the formalism of the light-front (LF) relativistic quantum mechanics it is possible to encode all the nonperturbative QCD effects in a LF quark model wave function $\psi\left(x, p_{t}^{2}\right)$ of a heavy hadron. The internal motion of a heavy $Q-$ quark inside the heavy flavor meson is described by the distribution function $\left|\psi\left(x, p_{t}^{2}\right)\right|^{2}$, which represents the probability to find a heavy quark carrying a LF fraction $\mathrm{x}=\mathrm{p}_{Q}^{+} / \mathrm{P}_{H_{Q}}^{+}$of the meson momentum and a transverse relative momentum squared $\mathrm{p}_{t}^{2}=\mathbf{p}_{t}^{2}$. In Ref. [7] this formalism has been used to establish a simple quantum mechanical relation between the inclusive semileptonic decay rate of the heavy hadron and that of a free heavy quark. In this paper we shall compute the preasymptotic effects for the inclusive semileptonic decays of the heavy baryons $\mathrm{H}_{Q}$ in the framework of the LF quark model, which is a relativistic constituent quark model based on the LF formalism.

\section{Basic formulae}

The approach of [7] relies on the idea of duality in summing over the final hadronic states. It has been assumed that the sum over all possible charm final states $X_{Q^{\prime}}$ can be modelled by the decay width of an on-shell $Q$ quark into an on-shell $Q^{\prime}$ quark folded with the $Q$-quark distribution function $f_{H_{Q}}^{Q}\left(x, p_{t}^{2}\right)=\left|\varphi_{H_{Q}}^{Q}\left(x, p_{t}^{2}\right)\right|^{2}$ The latter represents the probability to find a $Q$ quark carrying a LF fraction $x$ of the hadron momentum and a transverse relative momentum squared $p_{t}^{2}$. For the semileptonic rates the above mentioned relation takes the form

$\frac{d \Gamma_{S L}\left(H_{Q}\right)}{d t}=\frac{d \Gamma_{S L}^{Q}}{d t} R_{H_{Q}}(t)$

where $d \Gamma_{S L}^{Q} / d t$ is the free quark differential decay rate, $t=q^{2} / m_{Q}^{2}, q$ being the 4 -momentum of the $W$ boson, and $R_{H_{Q}}(t)$ incorporates the nonperturbative effects related to the Fermi motion of the heavy quark inside the hadron. The expression for $d \Gamma_{S L}^{Q} / d t$ for the case of non-vanishing lepton masses is given e.g. in [7]. $R_{H_{Q}}(t)$ in (11) is obtained by integrating the bound-state factor $\omega(t, s)$ over the allowed region of the invariant hadronic mass $M_{X_{Q^{\prime}}}\left(Q^{\prime}=\mathrm{c}\right.$ or $\left.\mathrm{s}\right)$ for the underlying transitions $Q \rightarrow Q^{\prime}$ :

$$
\begin{aligned}
& R_{H_{Q}}(t)=\int_{s_{\min }}^{s_{\max }} d s \omega_{H_{Q}}(t, s), \\
& \text { where } s=M_{X_{c}}^{2} / m_{b}^{2} \text { and } \\
& \begin{aligned}
\omega_{H_{Q}}(t, s)=m_{Q}^{2} x_{0} \frac{\pi m_{b}}{q^{+}} \frac{|\mathbf{q}|}{|\tilde{\mathbf{q}}|} \\
\bullet \int_{x_{1}}^{\min \left[1, x_{2}\right]} d x\left|\varphi_{H_{Q}}^{Q}\left(x, p_{t}^{* 2}\right)\right|^{2} .
\end{aligned}
\end{aligned}
$$

In Eq. (3) $x_{0}=m_{Q} / M_{H_{Q}}, x_{1,2}=x_{0} q^{+} / \tilde{q}^{ \pm}$, where $q^{+}=q_{0}+|\mathbf{q}|$ is defined in the $H_{b}$ rest frame and $\tilde{q}^{ \pm}=\tilde{q}_{0} \pm|\tilde{\mathbf{q}}|$ are defined in the $Q$ quark rest frame. In Eq. (2) the region of integration is defined through the condition $x_{1} \leq \min \left[1, x_{2}\right]$, i.e. $s_{\min }=\rho=$ $\left(m_{Q^{\prime}} / m_{Q}\right)^{2}, s_{\max }=x_{0}^{-2}\left(1-x_{0} \sqrt{t}\right)^{2}$. For other details see [7].

The structure of Eq. (1) suggests that in the limit of heavy quarks with infinite mass (i.e., $m_{Q} \rightarrow$ $\infty$ and $m_{Q^{\prime}} \rightarrow \infty$ ) one has

$\int d q_{0} \omega\left(q^{2}, q_{0}\right)=1$.

which means that the total inclusive width of the hadron is the same as the total inclusive width at the free quark level. The corrections to the free-quark decay picture are mainly due to the difference between the quark mass $m_{Q}$ and the hadron mass $M_{H_{Q}}$ as well 
as to the primordial motion of the $Q$-quark inside $H_{Q}$. These non-perturbative corrections vanish in the heavy-quark limit $m_{Q} \rightarrow \infty$, but at finite values of the $b$-quark mass a new parton description of inclusive semileptonic decays, based on the constituent quark model, has been derived. Note that the quark mass $m_{Q}$ appearing in 3 is the constituent quark mass that differs from the pole quark mass $m_{Q \text {,pole }}$ that enters the OPE result for the inclusive widths. As a consequence, Eq.1. generally contains the correction to first order in $1 / m_{Q}$ expansion to the free quark decay width. In principle, it is always possible to redefine the constituent quark mass $m_{Q}$ in such a way that the $1 / m_{Q}$ correction is eliminated from the total semileptonic width in accordance with the general statement of the OPE method. This is completely analogous to the way used to eliminate $1 / m_{Q}$ corrections from the ACCMM model [8] and has been discussed within the considered approach in [9] for a particular case of the $B \rightarrow X_{s} \gamma$ decay width.

A priory, there is no connection between the equal-time (ET) wave function $w\left(\mathbf{k}_{i}\right)$ of a constituent quark model and LF wave function $\psi\left(x_{i}, p_{i t}^{2}\right)$. The former depends on the center-of-mass momenta $\mathbf{k}_{i}$, while the latter depends on the LF variables $x_{i}$ and $\mathbf{p}_{i t}$. However, there is a simple operational connection between ET and LF wave functions [10]. The idea is to find a mapping between the variables of the wave functions that will turn a normalized solution of the ET equation of motion into a normalized solution of the different looking LF equation of motion. That will allows us to convert the ET wave function, and all the labor behind it, into a usable LF wave function. This procedure amounts to a series of reasonable (but naive) guesses about what the solution of a relativistic theory involving confining interactions might look like.

We convert from ET to LF momenta by leaving the transverse momenta unchanged, $\mathbf{p}_{\mathbf{i t}}=\mathbf{k}_{\mathbf{i t}}$, and letting $x_{i}=\frac{p_{i}^{+}}{P^{+}}$, where $P^{+}=\sum_{i} p_{i}^{+}$. In the center-of-mass frame $P^{+}=M_{0}$, where $M_{0}$ is the free mass operator, $M_{0}=\sum_{i} \omega_{i}$ with $\omega_{i}=$ $\sqrt{p_{i t}^{2}+p_{i z}^{2}+m_{i}^{2}}$ and

$p_{i z}=\frac{1}{2}\left(x_{i}-\frac{p_{t}^{2}+m_{i}^{2}}{x_{i} M_{0}^{2}}\right) M_{0}$

The momentum fractions and the transverse momenta obey the conservation law

$x_{1}+x_{2}+x_{3}=1, \quad \mathbf{p}_{1 t}+\mathbf{p}_{2 t}+\mathbf{p}_{3 t}=0$
Now we obtain the LF wave function from

$\psi\left(x_{i}, \mathbf{p}_{i t}\right)=\frac{\partial\left(k_{1 z}, k_{2 z}, k_{3 z}\right)}{\partial\left(x_{1}, x_{2}, x_{3}\right)} \cdot w\left(\mathbf{k}_{1}, \mathbf{k}_{2}, \mathbf{k}_{3}\right)$,

The straightforward calculation yields [11]

$\frac{\partial\left(k_{1 z}, k_{2 z}, k_{3 z}\right)}{\partial\left(x_{1}, x_{2}, x_{3}\right)}=\frac{\omega_{1} \omega_{2} \omega_{3}}{x_{1} x_{2} x_{3} M_{0}}$

with

$\frac{\omega_{i}}{x_{i}}=\frac{1}{2}\left(M_{0}+\frac{p_{i t}^{2}+m_{i}^{2}}{x_{i}^{2} M_{0}}\right)$

It can be easily verified that

$$
\begin{aligned}
& \int_{0}^{1}\left[d x_{i}\right] \int\left[d p_{i t}\right]\left|\psi\left(x_{i}, \mathbf{p}_{i t}\right)\right|^{2} \\
& \bullet \delta\left(\sum_{i} x_{i}-1\right) \delta\left(\sum_{i} \mathbf{p}_{i}\right)=1
\end{aligned}
$$

where

$$
\begin{aligned}
{\left[d x_{i}\right] } & =d x_{1} d x_{2} d x_{3} \delta\left(\sum_{i} x_{i}-1\right) \\
{\left[d p_{i t}\right] } & =d^{2} p_{1 t} d^{2} p_{2 t} t d^{2} p_{3 t} t \delta\left(\sum_{i} \mathbf{p}_{i t}\right)
\end{aligned}
$$

provided

$$
\begin{aligned}
& \int \Pi_{i} d \mathbf{k}_{i}\left|w\left(\mathbf{k}_{1}, \mathbf{k}_{2}, \mathbf{k}_{3}\right)\right|^{2} \\
& \bullet \delta\left(\mathbf{k}_{1}+\mathbf{k}_{2}+\mathbf{k}_{3}\right)=1 .
\end{aligned}
$$

Wave functions made kinematically relativistic in this fashion are used to calculate the form factors of heavy-to-heavy and heavy-to-light exclusive transitions [12].

\section{The Kalman-d'Souza model}

As was mentioned above, we consider the problem of a constituent bound state formed by a heavy quark interacting with the lighter ones. Specifically, we consider the Kalman-D'Souza model for heavy baryons [13].

The constituent quark masses are

$$
\begin{aligned}
& m_{u}=m_{d}=0.23 \mathrm{GeV}, \quad m_{s}=0.605 \mathrm{GeV} \\
& m_{c}=1.961 \mathrm{GeV}, \quad m_{b}=5.637 \mathrm{GeV}
\end{aligned}
$$

We denote the light quarks (u,d, or $\mathrm{s}$ ) by the labels 1,2 and the heavy quark (c or b) by the label 3. For the cases of $\Lambda$ and $\Sigma$ baryons where two of three quarks 
have equal masses we introduce the Jacobi relative coordinates

$\rho=\frac{1}{\sqrt{2}}\left(\mathbf{r}_{1}-\mathbf{r}_{2}\right), \quad \lambda=\sqrt{\frac{2}{3}}\left(\frac{m_{1} r_{1}+m_{2} r_{2}}{m_{1}+m_{2}}-r_{3}\right)$

For the $\Lambda_{c}$ and $\Lambda_{b}$ baryons in the ground state, the predominant configurations $(\approx 95 \%)$ of the heavy baryon wave functions contain no excitations in $\rho$ and $\lambda$. Hence

$$
\begin{aligned}
& \Psi(\rho, \lambda) \approx\left(\frac{\alpha_{\rho}}{\sqrt{\pi}}\right)^{3 / 2}\left(\frac{\alpha_{\lambda}}{\sqrt{\pi}}\right)^{3 / 2} \\
& \bullet \exp \left(-\frac{1}{2} \alpha_{\rho}^{2} \rho^{2}\right) \exp \left(-\frac{1}{2} \alpha_{\lambda}^{2} \lambda^{2}\right),
\end{aligned}
$$

where the factors $\alpha_{\gamma}(\gamma=\rho, \lambda)$ are given by

$\alpha_{\gamma}=\alpha_{\gamma}^{(0)}\left(\frac{\mu_{\gamma}}{m_{1}}\right)^{\frac{1}{4}}$,

with

$\mu_{\rho}=2 \frac{m_{1} m_{2}}{m_{1}+m_{2}}, \quad \mu_{\lambda}=\frac{3 m_{3}\left(m_{1}+m_{2}\right)}{2\left(m_{1}+m_{2}+m_{3}\right)}$,

and

$\alpha_{\rho}^{(0)}=0.29 \mathrm{GeV}, \quad \alpha_{\lambda}^{(0)}=0.32 \mathrm{GeV}$.

In Table 1, we compare the theoretical predictions of the Kalman-D'Souza model with the experimental data.

Table 1. The theoretical and experimental masses of the heavy baryons and the parameters $x_{0}$, $\alpha_{\rho}$, and $\alpha_{\lambda}$ used in the calculations. The theoretical masses are taken from 13], while the experimental ones are taken from [14] (for $H_{c}$ and $\Lambda_{b}$ ) and from [15] for $\Xi_{b}$

\begin{tabular}{|c|c|c|c|c|c|}
\hline \hline$H_{Q}$ & $M_{t h}$ & $M_{\exp }$ & $x_{0}$ & $\alpha_{\rho}$ & $\alpha_{\lambda}$ \\
\hline \hline$\Lambda_{c}$ & 2.280 & $2.285 \pm 0.0006$ & 0.86 & 0.29 & 0.40 \\
\hline$\Sigma_{c}$ & 2.454 & $2.453 \pm 0.0006$ & 0.80 & 0.29 & 0.40 \\
\hline$\Xi_{c}$ & 2.461 & $2.466 \pm 0.0014$ & 0.80 & 0.29 & 0.40 \\
\hline$\Lambda_{b}$ & 5.640 & $5.624 \pm 0.009$ & 1.00 & 0.29 & 0.41 \\
\hline$\Sigma_{b}$ & 5.960 & & 0.95 & 0.29 & 0.41 \\
\hline$\Xi_{b}$ & 6.045 & & 0.93 & 0.29 & 0.41 \\
\hline \hline
\end{tabular}

We now introduce the charge conjugate momenta

$\mathbf{k}_{\rho}=\frac{1}{2}\left(\mathbf{k}_{1}-\mathbf{k}_{2}\right), \mathbf{k}_{\lambda}=\frac{m_{Q}\left(\mathbf{k}_{1}+\mathbf{k}_{2}\right)-2 m_{q} \mathbf{k}_{3}}{2 m_{q}+m_{Q}}$, then the momentum space wave function $\psi\left(\mathbf{k}_{\rho}, \mathbf{k}_{\lambda}\right)$ normalized according to (3) is given by

$$
\begin{aligned}
\psi\left(\mathbf{k}_{\rho}, \mathbf{k}_{\lambda}\right)= & \left(\frac{1}{\tilde{\alpha}_{\rho} \sqrt{\pi}}\right)^{3 / 2}\left(\frac{1}{\tilde{\alpha}_{\lambda} \sqrt{\pi}}\right)^{3 / 2} \\
& \bullet \exp \left(-\frac{\mathbf{k}_{\rho}^{2}}{2 \tilde{\alpha}_{\rho}^{2}}\right) \exp \left(-\frac{\mathbf{k}_{\lambda}^{2}}{2 \tilde{\alpha}_{\lambda}^{2}}\right)
\end{aligned}
$$

with

$\tilde{\alpha}_{\rho}=\frac{1}{\sqrt{2}} \alpha_{\rho}, \quad \tilde{\alpha}_{\lambda}=\sqrt{\frac{2}{3}} \alpha_{\lambda}$

\section{The heavy baryon light-front wave func- tions}

We denote $\mathrm{x}=\mathrm{x}_{3}, \mathrm{y}=\mathrm{x}_{1}, \mathbf{p}_{3}=\mathrm{p}_{Q}$, then

$$
\begin{aligned}
& \left|\varphi\left(x, \mathbf{p}_{t}\right)\right|^{2}=\frac{1}{8} \int_{0}^{1-x} d y \int d \mathbf{p}_{1 t} \\
& \bullet\left(M_{0}+\frac{\mathbf{p}_{Q t}^{2}+m_{Q}^{2}}{x^{2} M_{0}}\right)\left(M_{0}+\frac{\mathbf{p}_{1 t}^{2}+m_{1}^{2}}{y^{2} M_{0}}\right) \\
& \bullet\left(M_{0}+\frac{\left(\mathbf{p}_{Q t}+\mathbf{p}_{1 t}\right)^{2}+m_{2}^{2}}{(1-x-y)^{2} M_{0}}\right)\left|\psi\left(\mathbf{k}_{\rho}, \mathbf{k}_{\lambda}\right)\right|^{2}
\end{aligned}
$$

where

$$
\begin{aligned}
& k_{\rho}^{2}=\left(\mathbf{p}_{1}+\frac{m_{1}}{m_{1}+m_{2}} \mathbf{p}_{Q}\right)^{2} \\
& k_{\lambda}^{2}=\mathbf{p}_{Q}^{2}=\mathbf{p}_{t}^{2}+\frac{1}{4}\left(\mathbf{x M}_{0}+\frac{\mathbf{p}_{t}^{2}+m_{Q}^{2}}{\mathbf{x M}_{0}}\right)^{2}
\end{aligned}
$$

In Fig.1 we show the two dimensional plot of the distribution function $\mathrm{f}_{\lambda_{b}}^{b}\left(\mathrm{x}, \mathrm{p}_{t}^{2}\right)$. In Fig.2 are shown the one dimensional distribution functions $\mathrm{F}_{H_{Q}}(\mathrm{x}), \mathrm{x}=\mathrm{x}_{3}$.

$$
\begin{aligned}
F_{H_{Q}}(x)= & \int_{0}^{1} d x_{1} \int_{0}^{1} d x_{2} \int \prod_{i=1}^{3} d p_{i t} \\
& \bullet\left|\psi_{H_{Q}}\left(x_{i}, \mathbf{p}_{i t}\right)\right|^{2} \\
& \bullet \delta\left(\sum \mathbf{p}_{i t}\right) \delta\left(\sum_{i} x_{i}-1\right) .
\end{aligned}
$$

for $\mathrm{H}=\Lambda, \Xi$ and $\mathrm{Q}=\mathrm{b}, \mathrm{c}$.

As illustrated in Fig.2 the distributions $\mathrm{f}_{\Xi_{c}}$ and $\mathrm{f}_{\Xi_{b}}$ are modified by the presence of the s quark instead of $\mathrm{u}(\mathrm{d})$ one. In Fig. 3 we show the non-perturbative factor $\mathrm{R}_{H_{Q}}(\mathrm{t})$ for $\Lambda_{b}, \Xi_{b}, \Lambda_{c}$, and $\Xi_{c}$ baryons. 


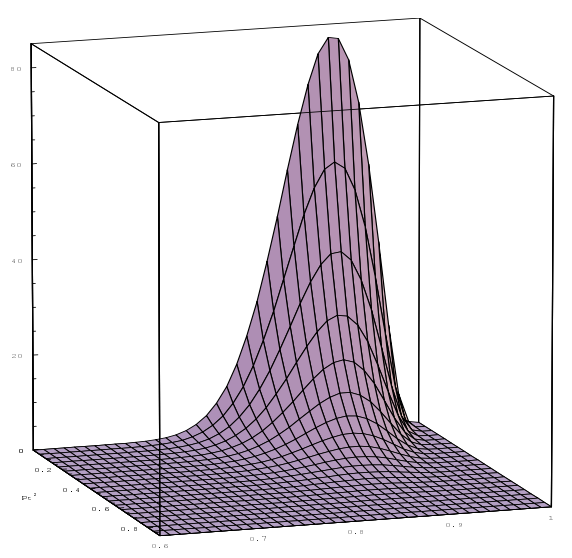

Figure 1. Two-dimensional distribution function $f_{\Lambda_{b}}^{b}\left(x, p_{t}^{2}\right)$.

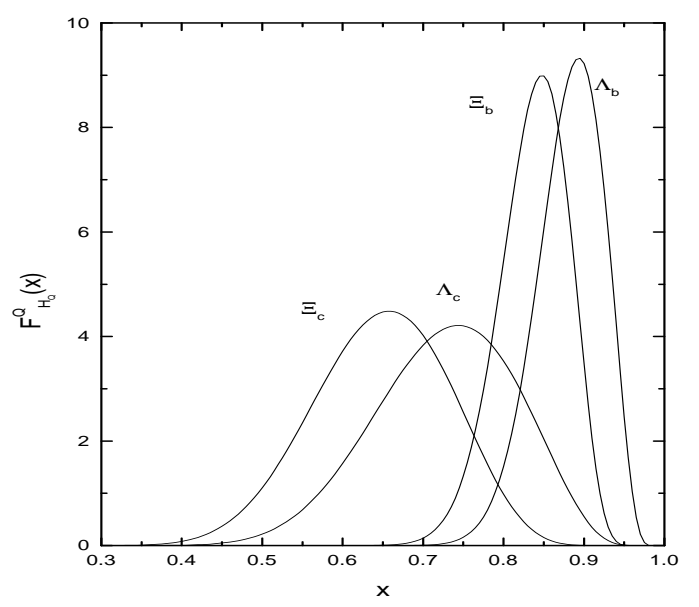

Figure 2. One-dimensional distribution functions $F_{H_{Q}}^{Q}(x)$ for $\Lambda_{c}$ and $\Lambda_{b}$ baryons.

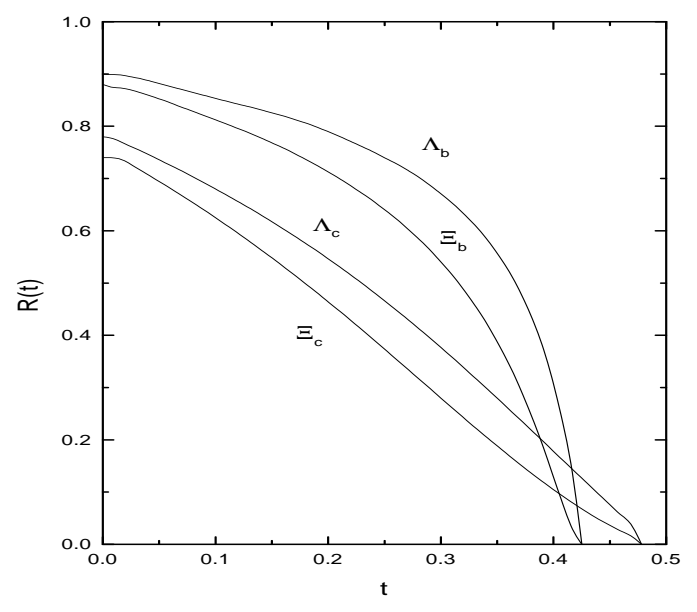

Figure 3. The bound-state factor $R_{H_{Q}}$ in Eq. (2).

\section{The results}

We have evaluated Eqs.(11-3) in case of the inclusive semileptonic decays $H_{Q} \rightarrow H_{Q^{\prime}} \ell \nu_{\ell}$ adopting the heavy quark distribution function $F_{H_{Q}}(x)$ from (25) with the Gaussian ansäts of the threequark wave function (15), that corresponds to the constituent quark model of ref. [13]. The parameters in (15) have been taken from Table 1. Our results for the SL decay widths of $H_{c}$ and $H_{b}$ baryons and electron branching ratios $\Gamma_{S L}\left(H_{Q}\right) / \Gamma^{t o t}\left(H_{Q}\right)$, where $\Gamma^{t o t}\left(H_{Q}\right)=1 / \tau\left(H_{Q}\right)$ is the total width of $H_{Q}$ baryon, are collected in Table 2 . For the lifetimes we take the values from the recent PDG publication [14] $\tau\left(\Lambda_{c}\right)=$ $0.206 \pm 0.012 \mathrm{ps}^{-1}, \tau\left(\Xi_{c}^{+}\right)=0.33_{-0.04}^{+0.06} \mathrm{ps}^{-1}$, $\tau\left(\Lambda_{b}\right)=1.229 \pm 0.08 \mathrm{ps}^{-1}, \tau\left(\Xi_{b}\right)=1.39 \pm 0.3 \mathrm{ps}^{-1}$.

For the $c$-flavored baryons, there exists only one inclusive value for the semileptonic branching ratio $\operatorname{BR}\left(\Lambda_{c} \rightarrow X_{s} e \nu\right)=4.5 \pm 1.7 \%$, the corresponding exclusive semileptonic branching $\operatorname{BR}\left(\Lambda_{c} \rightarrow \Lambda e \nu\right)$ being $2.0 \pm 0.6 \%$ 14]. For $\Xi_{c}^{+} \rightarrow \Xi^{0} e \nu$ there exists only exclusive branching ratio $2.3_{-0.9}^{+0.7} \%$. For the $b$-flavored baryons, there exists the semileptonic branching ratio $\operatorname{BR}\left(\Lambda_{b} \rightarrow \Lambda_{c} \ell \nu_{\ell}+\right.$ anything $)=7.9 \pm 1.9 \%$ which is dominated by the charm baryons. In Table 2 , in parentheses are also shown the results obtained using OPE with the pole quark masses $m_{c}=1.6 \mathrm{GeV}$, $m_{b}=4.8 \mathrm{GeV}$ and $\left|V_{c b}\right|=0.039$ [16]. We should mention a rather good agreement with the OPE predictions for the semileptonic rates in spite of the large 
difference of the constituent and pole quark masses that produces the large difference in $d \Gamma_{S L}^{Q} / d t \sim m_{Q}^{4}$. This difference is compensated by a factor $R\left(q^{2}\right)$ in Eq. (2).

Table 2. The predicted value of the inclusive semileptonic widths (in units of $\mathrm{ps}^{-1}$ ) and the corresponding branching ratios in $\%$. The experimental data are from [14]. $\left|V_{c b}\right|=0.04,\left|V_{s c}\right|=1.00$.

\begin{tabular}{|c|c|c|c|}
\hline \hline$H_{Q}$ & $\Gamma\left(X_{Q^{\prime}} e \nu_{e}\right)$ & $\Gamma\left(X_{Q^{\prime}} \tau \nu_{\tau}\right)$ & $B R\left(X_{Q^{\prime}} e \nu_{e}\right)$ \\
\hline \hline$\Lambda_{c}$ & $0.267(0.233)$ & & 5.50 \\
\hline$\Xi_{c}$ & $0.236(0.233)$ & & 7.79 \\
\hline$\Lambda_{b}$ & $0.086(0.070)$ & 0.019 & 10.57 \\
\hline$\Xi_{b}$ & $0.092(0.070)$ & 0.021 & 12.79 \\
\hline \hline
\end{tabular}

\section{Conclusions}

This is the most consistent quark model todate describing baryons in the sense that a single set of parameters is used for the whole spectra and then subjecting all of their properties namely the masses, radii and their wavefunction structures to a strict test by a decay model. The spectroscopy is successfully calculated using far less parameters than any other model 13. Decays of baryons are calculated [5]. Now in this paper we have successfully computed the inclusive semileptonic decays of the heavy baryons $H_{Q}$ by incorporating the LF formalism of [7].

\section{REFERENCES}

1. ALEPH Collaboration, D.Decamp et. al., Phys. Lett. B278 (1992) 209.

2. OPAL Collaboration, P.D.Acton et. al., Phys. Lett. B281 (1992) 394.

3. CDF Collaboration, F.Abe et. al., Phys. Rev. Lett. 77 (1996) 1439.

4. I.Bigi, M.Shifman, N.Uraltsev, Ann. Rev. Nucl. Part. Sci. 47 (1997) 591.

5. C. S. Kalman and B. Tran. Nuovo Cimento 104 (1991) 177.

6. A. Le Yaouanc, L. Oliver, O. Pène and J. C. Raynal. Phys. Rev. D9 (1974) 1415 , Phys. Rev. D11 (1975) 1272, Phys. Rev. D11 (1975) 2223. Hadron Transitions in the Quark Model, Gordon and Breach Science, N.Y. 1988.

7. S.Ya.Kotkovsky, I.M.Narodetskii, S.Simula, K.A.Ter-Martirosyan, Phys. Rev. D60 (1999) 114024.
8. I.Bigi et. al., Phys. Lett. B328 (1994) 421.

9. Y.-Y. Keum et. al., Phys. Lett. B471 (1999) 72.

10. F.Coester, Prog. Part. Nucl. Phys. 29 (1992) 1.

11. see e.g. F.Cardarelli, S.Simula, Phys. Lett. B421 (1998) 295.

12. I.L.Grach et. al., Phys. Lett. B385 (1996) 317; N.B.Demchuck et. al., Phys. Lett. B409 (1997) 435.

13. C.Kalman and I.D'Souza. Nuclear Physics B (Proc. Suppl.). 75B (1999) 3.

14. Review of Particle Physics, Euro. Phys. J. C15 (2000) 1.

15. UKQCD Collaboration, K.C.Bowler et. al., Phys. Rev. D54 (1996) 3641.

16. C.-Y.Cheng, Phys. Rev. D56 (1997) 2783. 